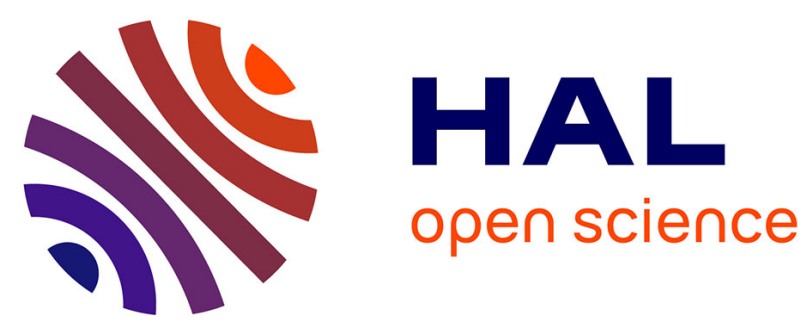

\title{
The Cost Optimal Solution of the Multi-Constrained Multicast Routing Problem
}

Miklós Molnár, Alia Bellabas, Samer Lahoud

\section{To cite this version:}

Miklós Molnár, Alia Bellabas, Samer Lahoud. The Cost Optimal Solution of the Multi-Constrained Multicast Routing Problem. [Research Report] 11004, Rennes 1. 2010, pp.17. inria-00529121

\section{HAL Id: inria-00529121 \\ https://hal.inria.fr/inria-00529121}

Submitted on 25 Oct 2010

HAL is a multi-disciplinary open access archive for the deposit and dissemination of scientific research documents, whether they are published or not. The documents may come from teaching and research institutions in France or abroad, or from public or private research centers.
L'archive ouverte pluridisciplinaire HAL, est destinée au dépôt et à la diffusion de documents scientifiques de niveau recherche, publiés ou non, émanant des établissements d'enseignement et de recherche français ou étrangers, des laboratoires publics ou privés. 


\title{
The Cost Optimal Solution of the Multi-Constrained Multicast Routing Problem
}

\author{
Miklós Molnár $^{*}$, Alia Bellabas ${ }^{* *}$, Samer Lahoud ${ }^{* * *}$ \\ miklos.molnar@lirmm.fr,alia.bellabas@irisa.fr,samer.lahoud@irisa.fr
}

\begin{abstract}
In this paper, we study the cost optimal solution of the well-known multi-constrained multicast routing problem. This problem consists in finding a multicast structure that spans a source node and a set of destination nodes with respect to a set of constraints. This optimization problem is particularly interesting for the multicast network communications that require Quality of Service (QoS) guarantees. Moreover, finding the multicast structure with respect to the defined QoS requirements while minimizing a cost function is an NP-difficult optimization problem. According to the state-of-the-art, to solve the multi-constrained multicast routing problem, most of the proposed algorithms search for a multicast tree. In this paper, we demonstrate that the optimal connected partial spanning structure that solves the multi-constrained multicast routing problem can be different from a partial spanning tree. Indeed, we show that the minimum cost solution always corresponds to hierarchy, a recently proposed simple generalization of the tree concept. For that, we define and analyze the partial minimum spanning hierarchies as optimal solutions for the multi-constrained multicast routing problem. Moreover, we propose a branch and cut type exact algorithm that is based on some relevant properties of the hierarchical solutions.
\end{abstract}

Key-words: Multicast, quality of service, multi-constrained Steiner problem, hierarchy, partial minimum spanning hierarchy

\section{Routage multi-contraint multicast optimal}

Résumé : Le routage multi-contraint multicast est un problème NP-difficile. Dans ce papier, nous étudions ce problème et analysons les propriétés de la solution optimale. Contrairement à ce qui est connu dans l'état de l'art, les arbres ne sont pas toujours les structures optimales pour le problème du routage multi-contraint multicast. Notre contribution principale est de définir la nature de la structure optimale pour le problème traité. Nous présentons les hierarchies qui sont des généralisations des arbres et nous prouvons que la solution optimale est toujours une hiérarchie. Nous analysons également les propriétés de la solution optimale et défissions un nouvel algorithme branch and cut pour trouver cette solution optimale.

Mots clés : Multicast, qualité de service, problème de Steiner multi-contraint, hiérarchie, hiérarchie minimiale de couvrement partial

\footnotetext{
Travail pour la nouvelle collection de publications internes de l'Irisa version électronique compatible avec les RR Inria

* Projet APR : LIRMM Monpellier

** Projet AtNet : équipe commune INSA et université de Rennes 1

*** Projet AtNet : équipe commune INSA et université de Rennes 1
} 


\section{Introduction}

Quality of Service QoS multicast routing known as multi-constrained multicast routing consists in constructing a multicast structure that spans a source node and a set of destination nodes. This multicast structure should meet a set of requirements such as delay, jitter, bandwidth, loss rate and cost, for each destination node. The multi-constrained multicast routing problem is known to be NP-difficult. Indeed, the most challenging QoS multicast routing techniques aim to support pointto-multipoint communications by 1) satisfying the QoS constraints and 2) leading to a reduction in the network resource consumption.

When there is one additive constraint to satisfy and no cost function to minimize, an efficient multicast routing can be achieved by forwarding packets over the shortest path tree. If the computed tree contains feasible paths from the source to each destination, the required QoS is assured, else there is no solution to satisfy the constraints for these destination nodes. To find a better solution within the feasible paths, a cost function can be introduced. The cost can be an arbitrary metric and independent from the QoS metrics. The minimum cost solution can minimize for instance the hop count.

The construction of the minimum cost multicast tree without QoS constraints corresponds to the Steiner Tree Problem. This problem is known to be NP-difficult [10]. Moreover, when a QoS constraint is present in the multicast routing problem and a minimum cost tree is required, the corresponding Constrained Steiner Problem is also NP-difficult as shown in [11]. Notice that most of the proposed works compute multicast trees that are partial spanning trees. However partial spanning trees do not always correspond to the optimal solution.

In this paper, we consider the general case of the constrained multicast routing with multiple QoS constraints. Moreover, we suppose that an additive cost function reflects the network usage that should be optimized. The two main reasons to couple optimization and multi-constrained routing are:

1. Real QoS requirements are often based on multiple constraints and the multicast structure must propose a feasible path for each destination.

2. The interest of the network operators and thus implicitly of the users, is to minimize network resource consumption.

In [16], the authors indicated that guaranteeing QoS and optimizing resource utilization are two conflicting interests and a trade-off should be achieved. Even if this conflict exists in some cases, we are interested in finding the best cost solution with respect to a set of QoS constraints. Thus, we investigate on the multi-constrained cost optimization of multicast structures. Our objective is to find the minimum cost spanning structure, where the end-to-end paths, from the source to the destinations, satisfy the considered QoS constraints. As it is demonstrated in [17], the multi-constrained multicast routing problems also known as multi-constrained partial spanning problems are NP-difficult even if there is only one destination.

In [13], the authors showed that a feasible partial spanning structure, that solves the multi-constrained spanning problem, may be different from a partial spanning tree. In this paper, our most important result is the exact characterization of the optimal solutions. We demonstrate that an earlier proposed simple generalization of the tree concept called hierarchy always corresponds to the minimum cost solution and can be used to describe all feasible solutions. Thus, we investigate on finding the partial minimum spanning hierarchy as the optimal solution of the multi-constrained partial spanning problem. The advantages to replace the spanning tree concept by the spanning hierarchy are conclusive: the hierarchies enable to define the optimal solution. Indeed, the optimal (minimum cost) multicast structure always correspond to a hierarchy.

The proposed multi-constrained partial minimum spanning hierarchy problem is NP-difficult. Since the hierarchies are different from trees, most of the known algorithms that are based on tree enumeration and node enumeration, to find optimal trees, can not be applied to find the cost optimal hierarchy. Therefore, we propose a branch and cut exact algorithm to solve this problem by considering an appropriate solution structure. To the best of our knowledge, our study is the first to formulate the optimal multicast routing problem using the hierarchy concept.

To appropriately present our contributions, this paper is organized as follows. Section 2 specifies the multi-constrained partial spanning problems for multicast QoS routing and provides an overview of the previously proposed approaches to solve them. Section 3 presents the hierarchy concept generalizing spanning trees and gives some general properties of the hierarchies. In Section 4, we prove that the optimal solution of the multi-constrained partial spanning problem is always a hierarchy. The hardness of the problem is discussed, and relevent properties of the multi-constrained minimal cost partial spanning hierarchies are presented. These properties are useful to design an efficient branch-and-cut exact algorithm described in Section 5.

\section{Problem Formulations and Related Works}

At the routing level, several multicast multimedia applications require multi-constrained multicast structures. In the literature, different objectives have been targeted and various solutions have been proposed. In this section, we present an overview of the most pertinent formulations of the addressed problem as well as a brief presentation of the different proposed 
route computation algorithms. To solve the addressed problem, we argue that the best solution corresponds to a minimum cost multicast structure with respect to the QoS constraints. Therefore, we propose the exact formulation of the minimum cost multi-constrained multicast routing problem.

\subsection{Problem Formulations}

Let $G=(V, E)$ be the undirected graph representing the network topology, where $V$ is the set of nodes and $E$ is the set of edges. The source node and the multicast destination node set are denoted by $s \in V$ and $D=\left\{d_{j} \in V, d_{j} \neq s, j=1, \ldots, r\right\}$ respectively. Each edge $e \in E$ is associated with $m$ QoS weights given by a weight vector $\vec{w}(e)=\left[w_{1}(e), w_{2}(e), \ldots, w_{m}(e)\right]^{T}$. The end-to-end QoS requirements i.e., constraints from the source to the destinations, are given by an $m$-dimensional constraint vector $\vec{L}=\left[L_{1}, \ldots, L_{m}\right]^{T}$. The QoS metrics can be roughly classified into additive such as delay, multiplicative such as loss rate or bottleneck such as available bandwidth. As it is explained in [13], bottleneck metrics can easily be dealt with by pruning from the graph all links that do not satisfy the QoS constraints, while the multiplicative metrics can be transformed into additive metrics by using their logarithm. Therefore, and without loss of generality, we only consider the additive metrics. The weight of a path $p\left(s, d_{j}\right)$ corresponding to the metric $i$ is given by $w_{i}\left(p\left(s, d_{j}\right)\right)=\sum_{e \in p\left(s, d_{j}\right)} w_{i}(e)$. Thus, a path $p\left(s, d_{j}\right)$ is feasible if:

$$
w_{i}\left(p\left(s, d_{j}\right)\right)=\sum_{e \in p\left(s, d_{j}\right)} w_{i}(e) \leq L_{i}, \text { for } i=1, \ldots, m
$$

Unicast $Q o S$ routing consists in finding a feasible path $p\left(s, d_{j}\right)$, between a source node $s$ and a destination node $d_{j}$. Often, a cost function $c$ evaluating the path should be minimized:

$$
\min \sum_{e \in p\left(s, d_{j}\right)} c(e)
$$

The multi-constrained QoS routing with a cost function, even in the unicast case is known to be NP-difficult as shown in $[17]$.

Multicast QoS routing aims to find a multicast structure $M(W, F)$, where $W$ is a set of nodes that can be present several time, and $F$ a set of edges, which can contain several times the same edge. The multicast structure $M(W, F)$ must contain at least one feasible path $p\left(s, d_{j}\right)$ from the source node $s$ to each destination $d_{j}, j=1 \ldots, r$. The optimal routing problem also aims to minimize the cost and thus avoids useless redundancies. The cost minimization is a good way to take into account the economical aspects of network usage.

Indeed, to solve the QoS multicast routing problem, most of the existing works are focusing on finding a partial spanning tree. Hereafter, we will show that a spanning tree cannot always solve the addressed problem.

Compared to the well known Steiner problem [10], where the minimum cost partial spanning tree is required, the construction of partial spanning structures satisfying multiple QoS requirements is even more complex.

To facilitate the comparison of the multi-constrained QoS paths, and to express the satisfaction of the QoS requirements, a non-linear scalar length function has been introduced in [13]. The length of a path $p\left(s, d_{j}\right)$ is defined as:

$$
l\left(p\left(s, d_{j}\right)\right)=\max _{i=1, \ldots, m}\left(\frac{w_{i}\left(p\left(s, d_{j}\right)\right)}{L_{i}}\right)
$$

If all of the constraints are satisfied, trivially $l\left(p\left(s, d_{j}\right)\right) \leq 1$. Thus, a feasible path can be also defined as a path whose length is less than 1.

To define the multi-constrained multicast routing problem, we propose the following formulation.

Problem 1. The problem of Multi-Constrained Minimum Cost Multicast (MCMCM) deals with finding the structure $G_{M}^{*}$ with minimum cost $c\left(G_{M}\right)$, containing at least one path $p\left(s, d_{j}\right)$ from the source node $s$ to each destination $d_{j} \in D$ that satisfies the given constraint vector $\vec{L}$, and given by the following Pareto dominance:

$$
\vec{w}\left(p\left(s, d_{j}\right)\right) \stackrel{d}{\leq} \vec{L} \text { and } c\left(G_{M}\right) \text { is minimum. }
$$

The cost function $c$ can be the frequently used hop-distance function or any other positive additive cost expressed on the edges. As we will see hereafter, this formulation is more appropriate than the proposed ones in the literature. In [13], three formulations have been proposed.

Problem 2. The Multiple Constrained Multicast (MCM) problem aims to find a partial spanning structure $G_{M}=(W, F), W \subseteq$ $V$ and $F \subseteq E$ that contains a path $p\left(s, d_{j}\right)$ from the source $s$ to each destination $d_{j} \in D$ such that:

$$
w_{i}\left(p\left(s, d_{j}\right)\right) \leq L_{i}, i=1, \ldots, m
$$




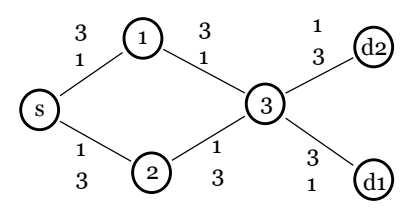

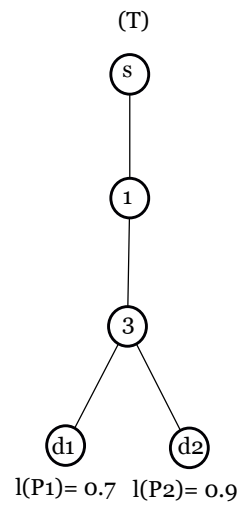

(a)

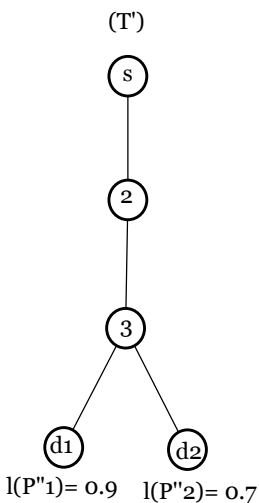

(b)
(G')

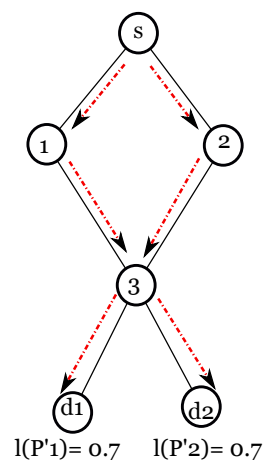

(c)

Figure 1: Relevance of the length function choice

or by using the Pareto dominance:

$$
\vec{w}\left(p\left(s, d_{j}\right)\right) \stackrel{d}{\leq} \vec{L}
$$

A trivial solution can be found by computing a set of feasible paths: one feasible path from the source to each destination if such a path exists.

Problem 3. In the Multiple Parameter Steiner Tree (MPST) problem, a tree $T_{M}=(W, F),\{s, D\} \subseteq W$ that minimizes a length function $l_{\text {multicast }}\left(T_{M}\right)$, is required.

This length can be an arbitrary vector norm. Typically, the used non-linear length function in [13] is given by:

$$
l_{\text {multicast }}\left(T_{M}\right)=\max _{i=1, \ldots, m} \frac{\sum_{e \in T_{M}} w_{i}(e)}{L_{i}}
$$

Notice that this non-linear length $l_{\text {multicast }}$ of a tree $T_{M}$ is not appropriate to characterize the quality of the multiconstrained multicast service at the destinations as detailed in the example given after Problem 4.

Problem 4. The Multiple Constrained Minimum Weight Multicast (MCMWM) problem combines the two previous objectives. The goal is to find a partial spanning structure $G_{M}=(W, F)$ that contains a feasible path to each destination $d_{j} \in D$ such that the length $l_{\text {multicast }}$ of $G_{M}$ is minimal:

$$
\vec{w}\left(p\left(s, d_{j}\right)\right) \stackrel{d}{\leq} \vec{L} \text { and } l_{\text {multicast }}\left(G_{M}\right) \text { is minimum }
$$

In the last two formulations, when the minimization of the total non-linear length of the partial spanning structure is proposed, we detect a limitation in the used length function $l_{\text {multicast }}$, which can considerably affect the quality of the computed structure. Indeed, we notice that this non-linear length function deduced based on the global consideration of the computed partial spanning structure does not consider the quality of each of the end-to-end paths. To illustrate this limitation we propose the example presented in Figure 1.

In Figure 1, the link weights are indicated and the QoS constraints are given by: $\vec{L}=[10,10]^{T}$. For the multicast group $\left\{s,\left\{d_{1}, d_{2}\right\}\right\}$, we notice that there are three sets of paths, to reach the two destinations. A tree $T$ with length $l_{m u l t i c a s t}(T)=1$, illustrated in Figure 1.(a), another tree $T^{\prime}$ with length $l_{\text {multicast }}\left(T^{\prime}\right)=1$, illustrated in Figure 1.(b), and a partial spanning structure $G^{\prime}$ with length $l_{\text {multicast }}\left(G^{\prime}\right)=1.2$, illustrated in Figure 1.(c). The solution of Problem 4 will be the tree $T$, or the tree $T^{\prime}$. However, if we consider the end-to-end quality of computed paths, we notice that the length $l\left(P_{1}^{\prime}\right)$ in $G^{\prime}$ is smaller than $l\left(P_{1}\right)$ in $T$, and $l\left(P_{2}^{\prime}\right)$ in $G^{\prime}$ and $l\left(P_{2}\right)$ in $T$ are equal. Thus, the structure $G^{\prime}$ is better considering the requirements of each of the destinations. 
We can conclude that the non-linear length of the unicast paths and the total length of the generated structure are not obviously correlated. Indeed, from $G^{\prime}$ to $T$ (or $T^{\prime}$ ), the total length of the partial spanning structure decreases while the length of the unicast paths increases.

Note that the aim of the routing is to find a structure containing at least one feasible path from the source to each destination, while minimizing the network resource usage expressed by an adequate cost function. For instance, the cost is a typically additive metric, which can be dependent or independent from the $m$ QoS metrics [5].

To characterize the relevance (the satisfaction of the QoS constraints) of the multicast structure, the most critical value of the QoS metrics can be used as the diameter of the multicast route. In this way, we can also define the multi-constrained multicast routing problem as follows.

Problem 5. The problem of Multiple Constrained Minimum Diameter Multicast (MCMDM) aims to find a structure G $G_{M}^{*}$ formed by feasible paths $p\left(s, d_{j}\right)$ from the source to each destination $d_{j} \in D$, while minimizing the non-linear diameter given by:

$$
D\left(G_{M}^{*}\right)=\max _{(j=1, \ldots, r)}\left(l\left(p\left(s, d_{j}\right)\right)\right.
$$

The non-linear diameter $D$ evaluates the critical quality of the computed structure $G_{M}^{*}$ based on the end-to-end proprieties of its paths. Indeed, the non-linear diameter of $G_{M}^{*}$ exactly corresponds to the non-linear length of the most critical unicast path. If the most critical destination is satisfied, the other destinations are also satisfied.

All of the presented spanning problems with multiple constraints are NP-difficult. For the three multicast routing problems presented in [13], the proof can be found in the same paper.

Lemma 1. The Multi-Constrained Minimum Cost Multicast (MCMCM) and the Multiple Constrained Minimum Diameter Multicast (MCMDM) problems are NP-difficult.

Proof. Since the multi-constrained minimum cost unicast routing is NP-difficult, the proof is trivial supposing only one destination.

As concluded in [13], the solution of the MCM and MCMWM problems is not always a tree. The solution corresponds to a partial spanning structure, which contains a feasible path to each destination. It also contains cycles that can not be eliminated. The solution of the minimum cost constrained partial spanning problem may also contain cycles in the set of the feasible paths. Here we raise the following important question: What kind of structure corresponds to the optimal solution of the MCMWM and MCMCM problems? The characterization of the solution allows to design exact algorithms and efficient heuristics to compute this solution.

\section{$2.2 \quad$ Related Works}

The multi-constrained multicast routing problem corresponding to the Multi-Constrained Partial Spanning Problem is known to be NP-difficult. To solve this problem, the different proposed approaches can be divided in three main classes, according to the objectives and the interest of each class of problems: (i) the first class aims to compute a spanning tree that minimizes a given cost function without checking the feasibility of the solution, (ii) the second class computes a minimum spanning tree, with respect to a set of QoS constraints, (iii) and the third class computes a spanning hierarchy (see the definition in the next section), with respect to a set of QoS metrics.

Notice that in the two first classes of problems, the different proposed solutions aim to compute spanning trees. There are two reasons for basing multicast structures on multicast trees (i) the data can be transmitted in parallel to various destinations along the tree links and (ii) the tree structure avoids redundancies.

We also notice that, if the addressed multi-constrained multicast problem has no cost function to minimize, any efficient multi-constrained unicast algorithm can be used, such as SAMCRA proposed in [21] and H_MCOP proposed in [12]. Therefore, we mainly focus the related works overview on the three above cited classes of problems.

In the first class of propositions, the solutions aim to minimize the cost of the multicast tree. The basic problem is known as the Steiner or Partial Minimum Spanning Tree (PMST) Problem. This NP-difficult problem has solicited a lot of interest, and a large number of exact [8] and heuristic [20] algorithms were proposed in the literature (cf. [23] [10] for two good overviews). The most known exact algorithms are based on the enumeration of the Steiner nodes ${ }^{1}$ and Steiner trees, and are called Steiner Tree Enumeration Algorithms. The first exact algorithm was proposed in [8]. Among the proposed heuristics, we can cite the Takahashi and Matsuyama algorithm proposed in [20]. This algorithm uses shortest paths to connect iteratively the destinations to the spanning tree.

In the second class, the addressed problems are known as the Constrained Steiner Tree (CST) Problems that are also NP-difficult [11]. To solve them, many of the proposed algorithms consider one QoS constraint as in [2], where the authors propose a branch and bound algorithm by using the Lagrangean Relaxation and heuristics to get lower and upper bounds

\footnotetext{
${ }^{1} \mathrm{~A}$ Steiner node is a node that has degree bigger than 2 in the spanning tree.
} 
in the branch and bound tree. However, most of the proposed algorithms for the CST problem are heuristics and generally consider the end-to-end delay. In [11], the authors proposed heuristic algorithms that construct a low cost spanning tree, with respect to a bounded delay on each multicast destination. The proposed algorithm computes a delay-constrained closure ${ }^{2}$ graph over the multicast group. Then, the algorithm constructs a constrained spanning tree of the closure graph using the well known Prim's algorithm [26]. Finally, the algorithm replaces the links in the spanning tree by the originally computed paths, and removes the generated loops. In [14], the authors also proposed an algorithm that approaches the minimum cost spanning tree solution with respect to the delay constraint. For that, the algorithm constructs two routing trees: a shortest path tree and an approached Steiner tree. Then, it identifies a given number of destinations $k$, where the difference between the delay observed in the Steiner tree and the delay in the shortest path tree for these destinations is large. For these destination nodes, their paths in the Steiner tree are replaced by their corresponding paths in the shortest path tree. The authors in [25] proposed a heuristic algorithm called The Bounded Shortest Multicast Algorithm (BSMA). This algorithm computes a least-delay tree, that spans the source node and the destination nodes. Then, it iteratively replaces the links that can be replaced by other links that reduce the total cost of the tree, without violation of the delay constraint, until the total cost of the tree can not further be reduced. The BSMA algorithm always finds a delay constrained tree, if one exists since it begins by the least-delay spanning tree.

When more than one QoS constraint are considered, the problem becomes more complex. In [22], the authors use the Lagrangean Relaxation Approach to construct special trees called LRATrees. Thus, the LRATree algorithm relaxes the constraints and constructs a new problem with one objective function to minimize $\left(\max _{\lambda} L(\lambda)=\min c\left(p\left(s, d_{j}\right)\right)+\right.$ $\sum_{i=1}^{r} \lambda_{i}\left(w_{i}\left(p\left(s, d_{j}\right)\right)-L_{i}\right)$, for each destination $\left.d_{j} \in D\right)$. $\lambda_{i}$ determines how much the violation of the $i^{\text {th }}$ constraint should be penalized. The algorithm computes the minimum cost path between the source node and every destination node, then combines these paths to obtain an initial feasible tree. This tree is updated when the Lagrangean parameter $\lambda=\left[\lambda_{1}, \ldots, \lambda_{m}\right]^{T}$ is adjusted as follows: $\lambda^{k+1}=\lambda^{k}+\theta^{k}\left(\vec{w}\left(p\left(s, d_{j}\right)\right)-\vec{L}\right)$, with $\theta^{k}=\frac{L\left(\lambda^{k+1}-\lambda^{k}\right)}{\left\|w_{i}\left(p\left(s, d_{j}\right)\right)-L_{i}\right\|^{2}}$. In [7], the author proposes an algorithm that searches for a feasible solution to the problem by finding, at first, a feasible partial tree that spans the source and some of the destinations. Then, it builds up the remaining destinations using a modified version of the H_MCOP algorithm [12]. This latter algorithm computes the shortest paths between two nodes by using the combined non-linear length function also presented in Equation 3.

However, a partial spanning tree may not always satisfy the required QoS constraints, while a set of unicast QoS paths can, and this considerably reduces the complexity of the addressed problem. Therefore, a trade-off between efficiency and complexity is needed.

In the literature, few proposed algorithms allow solutions that are different from spanning tree. The state of the art presents the Multicast Adaptive Multiple Constraints Routing Algorithm (MAMCRA) [13] as one of the most pertinent algorithms that attempts this trade-off even if there are several QoS constraints. In fact, the above cited algorithms aim to compute a tree as the only allowed solution, and this leads to the CST problem. MAMCRA is an algorithm that solves the multi-constrained multicast routing problem by computing a special routing structure. For this, MAMCRA proceeds in two steps:

- In the first step, the algorithm computes a set of optimal paths regarding a defined length function (cf. Equation 3). The computation of the shortest paths uses a slightly modified version of SAMCRA [21], an exact multi-constrained unicast algorithm.

- In the second step, MAMCRA tries to eliminate the useless redundancies that are produced in the first step. For this, MAMCRA uses a greedy algorithm. The greedy algorithm iteratively compares two paths that share at least one node and deletes the longuest prefix ${ }^{3}$ from the source node to the farest common node of one of them, if the resulted solution still feasible.

Furthermore, we notice that all of the above cited problems are mono-objective, with a cost or a length as the only objective. Considering the Multi-Objective Multicast Routing Problem, there are few proposed solutions as in[15][9], because of the reduced usefulness and the high complexity of this problem. Furthermore, most of the proposed solutions are based on meta-heuristics such as genetic algorithms [6] and ant colonies [19].

\section{$3 \quad$ Hierarchies as Spanning Structures in Graphs}

To identify the optimal solution of the multi-constrained partial spanning problem, we propose a brief overview of the hierarchy concept, which was proposed in [18].

\footnotetext{
${ }_{3}^{2} \mathrm{~A}$ closure graph on a set of nodes is a complete graph in which each link cost is equal to the shortest path between its nodes. 


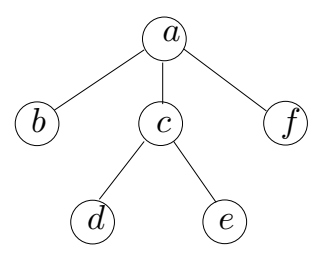

Figure 2: Example of a tree

Usually, spanning trees are considered as the minimum cost partial spanning structures. They correspond to connected sub-graphs without cycles. Indeed, if a minimum cost structure is required to solve a partial spanning problem without any constraint, this structure always corresponds to a spanning tree called the Steiner tree.

To solve the optimal multi-constrained multicast routing problem, some previous works proposed minimum length partial spanning trees. For instance, the solution of the MSTP problem presented in [13] corresponds to a partial spanning tree minimizing a non-linear length function. A minimum tree solution always exists but it does not always satisfy the end-toend constraints as it is stated in [13]. Trivially, the minimum length tree does not always contain a feasible path to each destination. Furthermore, real QoS multicast requests do not absolutely need a tree, but they mainly search for feasible paths from the source to all destinations, while minimizing the cost of the solution. Thus, if there is no tree satisfying the requests, the minimum cost solution can be a different structure containing a feasible path for each destination. For instance, the solution can be a set of feasible paths as it is the result of the first step of MAMCRA. Among the solutions offering feasible paths for all destinations, there is always an optimal solution with the smallest length (or cost), as it was formulated in MCMWM and MCMCM problems. Hereafter, we demonstrate that this optimal solution corresponds to a generalization of trees.

Trees are connected sub-graphs without cycles. In the following, a simple notation is used for rooted trees: the children of a node are enumerated between parenthesis after the parent node, as it is illustrated by the following expression corresponding to Figure 2:

$$
T=(a(b, c(d, e), f))
$$

In a rooted tree, each concerned node is present only once and has at most one parent node, except the root which has no parent.

The hierarchy concept was first introduced in [18], where tree-like structures containing node repetitions were defined. The following definition can be considered as the extension of the tree concept to define useful hierarchical structures related to graphs. In this paper, we only consider the rooted hierarchies. In fact, rooted hierarchies are more appropriate to describe the multi-constrained multicast routing problem.

Definition 1 (Rooted hierarchy). A non-empty rooted hierarchy in a graph is a connected structure, where each node occurrence has at most one parent node.

A rooted hierarchy can be given by the hierarchical tree-like enumeration of node occurrences, as illustrated in Figure 3.

$$
H=(a(c(d(f)), b(d(c, e))))
$$

A hierarchy is not necessarily exempt from repetitions: nodes and edges of the related graph may be present several times in a hierarchy. In our example, the nodes $c$ and $d$ are present twice. It is important to emphasize that a node can have many occurrences in a hierarchy called node occurrences, and this is also applied for edges and arcs. Since the different occurrences of the same element may play different roles in the hierarchy, the distinction and the identification of the occurrences is substantial. Indeed, a node occurrence can be an intermediate node in a hierarchy while another occurrence of the same node can be a leaf, like the node $c$ in Figure 3 (b). If a node occurrence has at least two children then it is called a branching node occurrence, like the node $d$ in Figure 3 (b). Notice that a hierarchy is not a sub-graph, but it generates a sub-graph in the basic graph. This sub-graph is called the image of the hierarchy in the basic graph. The image of the mentioned hierarchy in the basic graph is shown in Figure 3 (a).

Hierarchies can be directed or undirected. They also can span a set of nodes or all nodes. However, to describe the here analyzed single source QoS multicast routing problem, directed partial spanning rooted hierarchies can be applied. These hierarchies are directed from the source to the destinations. We will see later that the use of directed rooted hierarchies allows an accurate description of the solution for the multi-constrained partial minimum spanning problem.

The enumeration of the most important properties of the hierarchies facilitates their construction to solve the constrained spanning problem. 


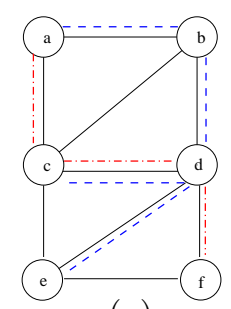

$(a)$

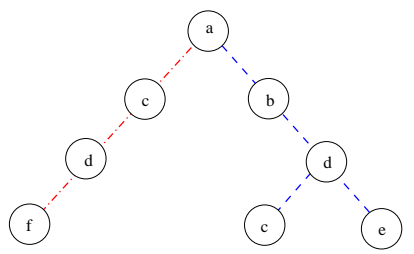

(b)

Figure 3: Example of an undirected rooted hierarchy in an undirected graph

\subsection{Basic Properties of Hierarchies}

Recall that trees are special hierarchies where each node has at most one occurrence. Thus, a tree is a hierarchy.

Notice that, some properties of trees are true for hierarchies but not all, whereas all properties characterizing hierarchies are true for trees. Some important properties of hierarchies can be formulated as follows.

- Since each node occurrence is different in a hierarchy, the cyclomatic ${ }^{4}$ number of a hierarchy is equal to 0 . However, the cyclomatic number of the image of a hierarchy can be different from 0 since the generated sub-graph can contain cycles.

- In a hierarchy, there is one and only one path between two node occurrences.

- A hierarchy may contain more than one node occurrence of a node and eventually more than one edge/arc occurrence of an edge/arc. Moreover, when the same node pair is repeated in a hierarchy, the arc delimited by these nodes is also repeated.

If a graph element is present several times, its occurrences should be distinguished. In the following, we will distinguish the occurrences of a node $x$ by different exponents $x^{1}, x^{2}, \ldots$ when needed.

\subsection{Hierarchies Used by MAMCRA}

The most efficient heuristic algorithm to solve the multi-constrained multicast routing problem is MAMCRA [13]. This algorithm that is briefly presented in Section 2 computes a set of optimal paths with eventual redundancies, then uses a greedy algorithm to eliminate some of these redundancies. At first, we propose a brief analysis of the structures used by MAMCRA. Notice that MAMCRA does not guarantee the optimal solution. We will see that the set of optimal paths returned in the first step of MAMCRA does not necessarily contain the paths belonging to the optimal solution neither of the MCMCM nor of the MCMWM problems. After the brief analysis of the paths proposed by MAMCRA, some important properties of the optimal minimum cost or minimum length solution are presented in the next section.

In the following, we will prove that the set of paths that are computed in the first step of MAMCRA as well as the final solution of MAMCRA are hierarchies.

Lemma 2. A set of paths from the same source node to different destinations corresponds to a hierarchy.

Proof. The lemma is trivial, since the set of paths is connected due to the common source node and each node occurrence has at most one parent in this set. Thus the first step of MAMCRA constructs a hierarchy.

Lemma 3. The multicast routing structure obtained after the second step of MAMCRA is a hierarchy.

Proof. The first step of MAMCRA computes a hierarchy. The second step eliminates some redundancies using the following operation. Let $p_{1}\left(s, x^{1}, d_{1}\right)$ and $p_{2}\left(s, x^{2}, d 2\right)$ be two paths sharing a common node $x$. Under some conditions as explained in [13], the part from $s$ to $x$ of one of the paths is omitted: for example $p_{1}\left(s, x^{1}\right)$ is deleted and the concatenation $p_{2}\left(s, x^{2}\right)+$ $p_{1}\left(x^{2}, d_{1}\right)$ is used for the destination $d_{1}$, if this new path still feasible. To prove our lemma, it is sufficient to demonstrate that the redundancy elimination algorithm does not change the structure: the obtained structure is also a hierarchy. Since $p_{2}\left(s, x^{2}\right), p_{1}\left(x^{2}, d_{1}\right)$ and $p_{2}\left(x^{2}, d 2\right)$ are paths, and they share only the node occurrence $x^{2}$, the children of $x^{1}$ will change their parent node to $x^{2}$, and they consequently still having one parent node. Therefore, MAMCRA returns a hierarchy.

\footnotetext{
${ }^{4}$ The cyclomatic number of a graph is defined as the number of independent cycles in the graph.
} 


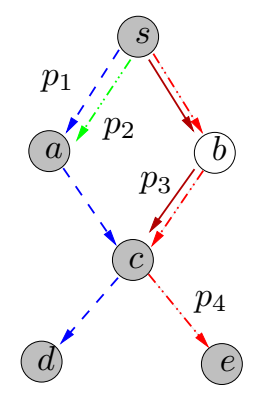

(a)

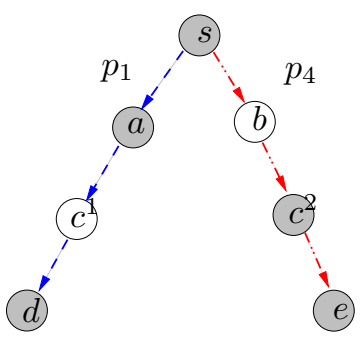

(c)

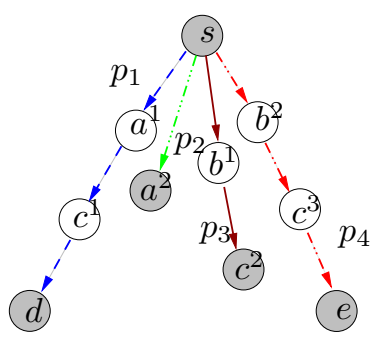

(b)

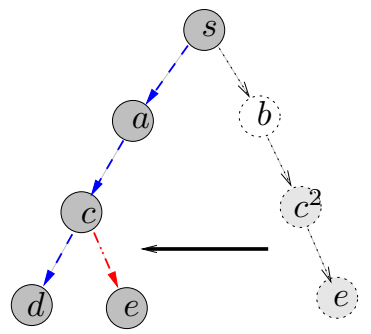

$(d)$

Figure 4: The hierarchies computed by MAMCRA

Figure 4 shows the evolution of the multicast structure computed by MAMCRA, where four destinations $a, c, d$ and $e$ should be spanned. Figure 4 (a) presents the four paths computed by SAMCRA in the basic graph, while Figure 4 (b) presents these paths as a hierarchy. This hierarchy enables to distinguish the different occurrences of the nodes that are shared by more than one path. As paths $p_{1}\left(s, a^{1}\right)$ and $p_{2}\left(s, a^{2}\right)$ have the same sequence of nodes, one of them can be omitted. Therefore, $a$ becomes an intermediate destination node in $p_{1}$. Similarly, the destination $c$ can be considered as an intermediate node in $p_{4}$. These simplifications are detailed in [13]. We can consider that the four destinations are spanned by two feasible shortest paths returned by the first step of MAMCRA as illustrated in Figure 4 (c). The destinations $a$ and $c$ are intermediate nodes in the paths $p_{1}$ and $p_{4}$ respectively. Moreover, another occurrence of the node $c$ is a relay node in the path $p_{1}$. According to Lemma 2, this set of paths corresponds to a hierarchy. Let us suppose, that the concatenation of $p_{1}\left(s, c^{1}\right)$ and $p_{4}\left(c^{2}, e\right)$ is a feasible path. In this case, the greedy algorithm in the second step of MAMCRA replaces the path $p_{4}$ by this concatenation as it is indicated in Figure $4(\mathrm{~d})$. The resulting structure is a hierarchy.

It is important to emphasize that there is no obvious correlation between the shortest paths and the optimal solution. Moreover, even if we use an exact algorithm to eliminate the redundancies, the resulted hierarchy may be different from the optimal solution.

Lemma 4. The minimum length solution of the MCMWM problem and the minimum cost solution of the MCMCM Problem do not necessarily belong to the set of shortest paths computed by the first step of MAMCRA using the non-linear length.

Proof. The proof is based on an example. Figure 5 illustrates that the shortest paths, considering the non-linear length, are not necessarily included in the optimal solutions. In the given graphs, the costs $c$ and the link weights $w$ are indicated. Let us suppose that $a$ is the source node, and there are two destinations: $b$ and $d$. The QoS constraints are given by $\vec{L}=[7,7]^{T}$. In Figure 5 (a), we show that the shortest paths $(a, b),(a, d))$, using the cost and the non-linear length function respectively, do not contain neither the minimum cost solution nor the solution with minimum non-linear length. These optimal solutions correspond to the tree $(a(c(b, d)))$.

Figure $5(\mathrm{~b})$ illustrates the fact that the tree with minimal non-linear length is not the optimal solution for the end-to-end constrained multicast routing problem. In this example, the optimal solution of the MCMWM problem is the tree $(a(c(b, d)))$. This tree has a greater non-linear length at the leaves than the tree $(a(b, d))$. Moreover, the solutions of both problems can be different. Indeed, the minimum cost solution does not necessarily correspond to the minimum length solution of MCMWM. 

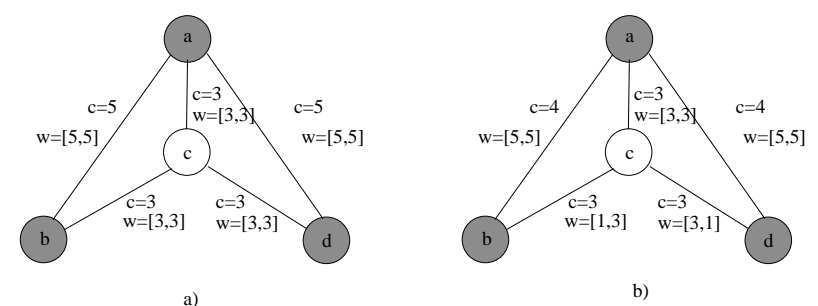

Figure 5: The set of shortest paths and the set of minimum cost paths does not contain the optimal solution

\section{The Minimum Partial Spanning Hierarchies to Solve the Multi-Constrained Multicast Routing}

It is known from [13] that some feasible multicast structures under multiple end-to-end constraints do not correspond to a spanning tree. It is the case with the solution returned by MAMCRA. However the exact structure of these solutions was not yet analyzed. The introduction of the hierarchies allows an accurate definition of these multicast structures. Moreover, our analysis aims to determine the optimal solution of the multi-constrained multicast routing. We are particularly interested in describing the optimal solution of Problem 1 (MCMCM). We mainly investigate on this minimum cost solution, whereas the spanning structures solving Problem 4 (MCMWM) have the same properties.

\subsection{The Minimum Cost Solution}

Let $M$ be the optimal (of minimum length or minimum cost) multicast structure, with respect to the given QoS constraints.

Theorem 1. The optimal multicast structure $M$ with respect to multiple constraints on positive additive metrics is always a directed partial spanning hierarchy.

Proof. The optimal multicast structure must contain at most one directed feasible path from the source to each destination. Consequently, this structure is directed and connected. The structure does not obligatorily span the entire node set, it is a partial spanning structure. It is sufficient to prove that the minimum cost partial spanning structure cannot contain any superfluous cycles. In other terms, each node occurrence has at most one parent node and one arc between this parent and the node occurrence in the computed structure.

Let us suppose that a node occurrence $v^{1}$ has two parent nodes (or two incoming arcs from the same parent node) in the minimum cost structure $M$ spanning $\{s\} \cup D$. Then one of the predecessor arcs of $v^{1}$ can be dropped without loss of the connectivity and the remained structure covers $\{s\} \cup D$. Therefore, $M$ cannot be the multicast structure with minimum cost.

In the following, we call this solution the multi-constrained minimum partial directed spanning hierarchy (abbreviated by MC-MPDSH). Using the hierarchy concept, the MCMCM problem can be re-formulated as follows.

The optimal (minimum cost) multi-constrained multicast routing that consists in finding a minimum cost structure having at most one directed path $p\left(s, d_{j}\right)$ from the source to each destination $d_{j} \in D$, with respect to the given constraints $\vec{w}\left(p\left(s, d_{j}\right)\right) \stackrel{d}{\leq} \vec{L}$ is equivalent to find the multi-constrained minimum partial directed spanning hierarchy with respect to the given constraints.

Similarly, the MCMWM problem can also be formulated using the hierarchy concept.

\subsection{Properties of the MC-MPDSH}

In the following, we enumerate some properties of the MC-MPDSH. This properties enable to i) better identify the optimal solution ii) demonstrate some limitations of known computational algorithms of spanning structure and iii) design more efficient exact and heuristic algorithms.

Thus, we investigate on the properties of the minimum cost solution. The following two properties are always true in the optimal multicast routing structure.

Property 1. The leaves in the optimal spanning hierarchy $M$ are destinations.

Proof. Let us suppose that $t \in M$ is a leaf node but it is not a destination. In this case, $t$ and its precedent arc can be dropped because all of the destinations are spanned with the remained connected structure. Therefore, $M$ can not be the minimum spanning structure. 


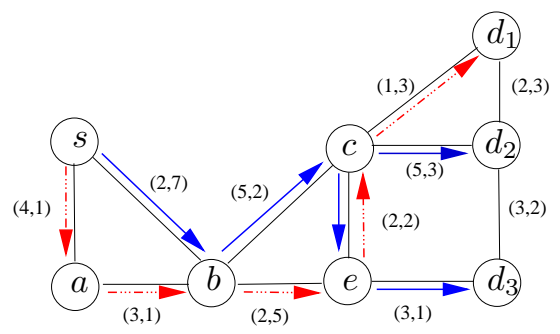

Figure 6: An edge can be used several times in an MC-MPDSH

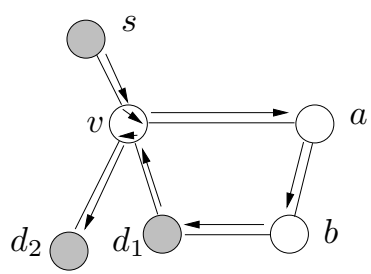

Figure 7: A node is present twice in a path

Notice that a destination may also correspond to an arbitrary intermediate node occurrence.

Property 2. In the optimal hierarchy $M$ the directed path from the source $s$ to an arbitrary node occurrence $v^{1}$ belonging to $M$ is a feasible path.

Proof. $M$ contains a feasible path for each destination. For $v^{1} \in D$, the property is trivial. Let us suppose that $v^{1} \notin D$ (and thus, after Property $1, v^{1}$ is an internal node of a path toward a destination leaf). Let us also suppose that the path $\left(s, v^{1}\right)$ is not feasible. Consequently, the paths from the source to the destinations which are extended from $v^{1}$ are not feasible because the metrics are positive and additive. This is in contradiction with the fact that $M$ contains a feasible path to all destinations.

We show that an edge/arc may belong several times to the solution. In our problem, the solution is supposed to be a directed hierarchy in an undirected graph. Figure 6 gives an example that an edge of the graph can be used by a hierarchy several times and in both directions. In this example, the edge $(c, e)$ is used three times in the optimal spanning hierarchy. This hierarchy is the only one providing feasible paths from the source $s$ to the destinations $d_{1}, d_{2}$ and $d_{3}$ when the QoS constraints are given by the vector $\vec{L}=[13,13]^{T}$. Let us notice that there are upper bounds on the number of usage of an edge and a node in the optimal hierarchy. In the following, our objective is to find these upper bounds to design efficient hierarchy computation algorithms.

According to Property 1, a leaf of the MC-MPDSH is always a destination. Therefore, the MC-MPDSH has at most $|D|$ leaves. The following three properties are not trivial in MC-MPDSHs, but they considerably help the construction of the optimal solution.

Property 3. In a directed path from the source $s$ to an arbitrary destination $d_{j}$ in the MC-MPDSH, a node is present at most once.

Proof. Let us suppose that a node $v$ is present twice in a directed path of the MC-MPDSH as it is illustrated in Figure 7. If there is a destination $d_{1}$ between the two occurrences of $v$, the last segment $\left(d_{1}, v^{2}\right)$ from $d_{1}$ to the second occurrence of $v$ can be eliminated, and the obtained hierarchy has a lower cost and better QoS values at the destination nodes. If there are several destinations in the cycle, trivially, the last segment from the last intermediate destination node occurrence to $v^{2}$ can be eliminated. If there is no destination, then the entire cycle can be deleted. Thus, the optimal solution can not contain two occurrences of a node in the same directed path from the source.

This property is also true regarding the source node itself: no directed path in an MC-MPDSH makes its possible to return to the source node. Consequently, the source is present only once in an MC-MPDSH. Let us notice that a node different from the source may be present several times in the optimal hierarchy but each occurrence of the node belong to a different path.

Property 4. The number of the occurrences of a node in the MC-MPDSH is upper bounded by $|D|$. 


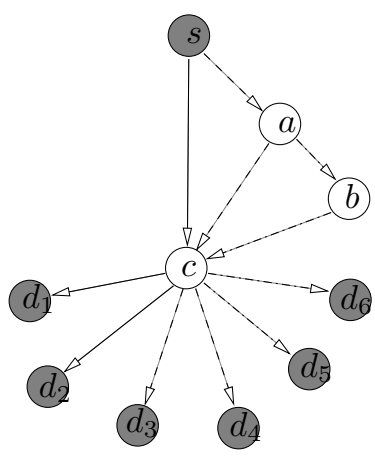

(a)

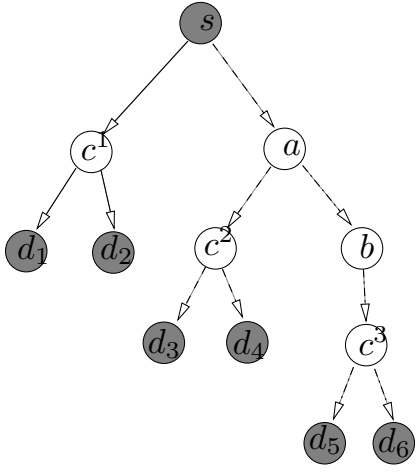

(b)

Figure 8: A maximum number of branching node occurrences of a node in the hierarchy

Proof. According to Property 3, a node is present at most once in any path of the MC-MPDSH. Let us suppose that the node $v$ is present in all of the directed paths of the MC-MPDSH. In the worst case, there are $|D|$ directed paths such that any path $\left(s, d_{j}\right)$ does not include any other destination $d_{k}$. Since $v$ can belong to all of them, the upper bound is trivialy equal to $|D|$.

If some occurrences of a node are branching nodes in the optimal hierarchy, then the number of its occurrences can be smaller than $|D|$.

Property 5. If a destination is a leaf node in the MC-MPDSH, then it has only one occurrence (the leaf occurrence) in the optimal solution.

Proof. Let us suppose that the node $d_{1}$ is present twice in the MC-MPDSH: a first node occurrence $d_{1}^{1}$ is a leaf in the path $p_{1}\left(s, d_{1}^{1}\right)$ and $d_{1}^{2}$ is another occurrence in an other path $p_{2}\left(s, d_{2}\right)$. Since the MC-MPDSH contains only feasible paths, the path $p_{2}\left(s, d_{2}\right)$ and its prefix $p_{2}\left(s, d_{1}^{2}\right)$ are both feasible. Thus, the path $p_{1}\left(s, d_{1}^{1}\right)$ is useless for $d_{1}$, and at least the last arc of this path can be deleted without affecting the feasibility of the solution. If there are intermediate destinations in $p_{1}\left(s, d_{1}^{1}\right)$, then only the last segment from the last destination to $d_{1}^{1}$ can be deleted. Therefore, the two occurrences of $d_{1}$ are not possible in the optimal solution.

Property 6. In an $M C-M P D S H$, a node can have at most $\left\lfloor\frac{|D|}{2}\right\rfloor$ branching node occurrences.

Proof. The maximal number of branching node occurrences of a given node $v$ is produced when all of the branching node occurrences correspond to $v$ and each occurrence is a common node of at most two paths toward two destination leaves. Note that there are at most $|D|$ leaves in the hierarchy. If $|D|$ is even, the number of branching node occurrences of $v$ is $\frac{|D|}{2}$. Else if $|D|$ is odd, the last occurrence of $v$ is not a branching node, since it contains only one successor. Therefore, the upper bound of the branching node occurrences of $v$ is $\left\lfloor\frac{|D|}{2}\right\rfloor$.

Figure 8 illustrates the worst case introduced in Property 6. The last two properties can be generalized in Property 7 .

Property 7. Let $v^{i}$ be the $i^{\text {th }}$ occurrence of the node $v$ in a sub-hierarchy $H$ in an MC-MPDSH and $d_{H}^{+}\left(v^{i}\right)$ its out-degree. Let $l_{H}$ be the number of leaves in $H$. For the occurrences of the same node $v$, the following inequality always holds:

$$
\sum_{v^{i} \in H} d_{H}^{+}\left(v^{i}\right) \leq l_{H}
$$

Proof. If $v$ has a leaf occurrence in $H$, then according to Property 5, this occurrence is the only one of the node $v$ in $H$, and $v$ should be a destination. In this case, Property 7 is trivial.

If the different occurrences of $v$ are not leaves, then each node occurrence has at least one child. More precisely, the occurrence $v^{i}$ has $d_{H}^{+}\left(v^{i}\right)$ sub-hierarchies. In each sub-hierarchy there is at most one leaf node. According to Property 3 , each path to a leaf contains at most one occurrence of $v$.

Note that the metrics in the graph are positive and additive. Supposing an optimal solution for a given QoS request, what can be stated about the sub-optimality of the sub hierarchies of the optimal hierarchy? The following property enables to establish an important relation between the QoS constraints and gives a sub-optimality property. 
Property 8. Let $M$ be an MC-MPDSH rooted at s and satisfying the constraints $\vec{L}$ in the leaves. Let $M_{v}$ be a sub-hierarchy of $M$ rooted at $v \neq s$. Let $\vec{w}(p(s, v))$ be the weight vector of the path $p(s, v)$. The sub-hierarchy $M_{v}$ is an $M C$ - $M P D S H$ from $v$ to the destination occurrences that it contains with respect to the constraints $\overrightarrow{L_{v}}=\vec{L}-\vec{w}(p(s, v))$.

Proof. A destination node has only one destination occurrence in the MC-MPDSH (other occurrences of the node can be used as intermediate relay node occurrences toward other destinations but the node should receive multicast messages as a destination only once). Therefore, we refer to destinations by destination occurrences.

At first, we prove that $\vec{w}\left(p\left(v, d_{j}\right)\right) \stackrel{d}{\leq} \overrightarrow{L_{v}}=\vec{L}-\vec{w}(p(s, v))$ for all destinations $d_{j} \in M_{v}$. Let us suppose that for the destination $d_{j} \in M_{v}$ the weight vector $\vec{w}\left(p\left(v, d_{j}\right)\right)$ does not dominate $\overrightarrow{L_{v}}$. In this case, $\vec{w}\left(p\left(s, d_{j}\right)\right)$ does not dominate $\vec{L}$ and thus $M$ can not be feasible.

Secondly, we prove that $M_{v}$ is the minimum cost hierarchy in the set of the feasible hierarchies spanning the destinations in $M_{v}$. Let us suppose that $M_{v}$ is not a minimum cost solution but only a feasible solution for the partial problem. In this case, there is an MC-MPDSH $\tilde{M}_{v}$ rooted at $v$ the cost of which is less then the cost of $M_{v}$. By replacing $M_{v}$ by $\tilde{M}_{v}$ in the hierarchy $M$, we obtain a feasible spanning hierarchy with less cost, which is in contradiction with the fact that $M$ is an MC-MPDSH.

To our knowledge, our study is the first one to analyze the minimal solution of the multi-constrained partial spanning problem corresponding to the QoS multicast routing problem. To solve this problem, the design of exact or heuristic algorithms is an important challenge for the QoS multicast routing.

\section{An Exact Algorithm to compute the Multi-Constrained Minimum Partial Spanning Hierarchies}

As it was presented earlier, the multi-constrained multicast routing problem is NP-difficult. Unfortunatly, the techniques which are known to solve the Steiner problem such as the Steiner Tree Enumeration Algorithms and the Topology Enumeration Algorithms can not be applied here, because of the multiple occurrences of the graph elements in the optimal solution. Precisely, the solution we try to compute is not a sub-graph but a hierarchy.

The branch and cut algorithm is a good candidate to exactly solve the addressed problem. Furthermore, the use of the cut operations corresponding to the optimal hierarchy properties are interesting to accelerate the computation. In the following, we present our first proposal to compute the optimal MC-MPSH. We argue that several exact algorithms can be found and further research is needed to find more efficient solutions.

\subsection{A Branch and Cut Algorithm}

The use of the branch and cut algorithm allows the enumeration of the directed partial spanning hierarchies satisfying the limitations mentioned in Section 4.2. The here proposed algorithm selects hierarchies in an increasing order of their total cost. The first hierarchy in the course of the enumeration, which covers all destinations and satisfies the end-to-end constraints, corresponds to the optimal solution. Each node of our virtual search tree corresponds to a directed hierarchy rooted at the source node. The algorithm begins with a hierarchy only containing the source node $s$. The cost and the end-to-end weights on the paths from the source to the leaves are computed. Trivially, a hierarchy is not feasible if it contains a leaf which does not satisfy the given QoS constraints. At each step of the branch and cut algorithm, the hierarchies corresponding to the leaf nodes of the search tree are examined and the hierarchy with the lowest cost is selected. If there is no leaf hierarchy in the search tree satisfying the end-to-end constraints, the problem has no solution in the given graph with the given constraints. Let $K$ be the set of leaves of the search tree. The meta-code of the algorithm is given by Algorithm 1 .

The crucial question of this algorithm is how the valid successors of the selected hierarchy $H$ can be computed. These successors are the hierarchies enlarged only by adjacent arcs from the leaves of $H$ that satisfy the QoS constraints. This kind of arcs are called fringe arcs, expression borrowed from [24]. The fringe arcs of a hierarchy are illustrated by Figure 9.

Lemma 5. Algorithm 1 enumerates all valid spanning hierarchies in increasing order. This enumeration does not loss any valid hierarchy.

Proof. Notice that for a given valid hierarchy $H$, Algorithm 1 adds fringe links to the leaves of $H$ and constructs all valid hierarchies by combining the added links. Indeed, for $k$ added fringe links the algorithm constructs at most $\sum_{i=1}^{k}\left(\frac{k !}{i !(k-i) !}\right)$ new valid hierarchies. Let us suppose that the algorithm omits to construct a valid $H^{\prime}$ that contains an arc $a$ added at an intermediate node of $H$ and $H^{\prime}$ is valid. In the search tree of Algorithm 1 there is a node $v$ in a hierarchy $H^{\prime \prime}$ for which the arc $a$ is a fringe arc. Since $H^{\prime}$ is valid, the successor of $H^{\prime \prime}$ containing $a$ is valid and the algorithm has already enumerated this hierarchy. 

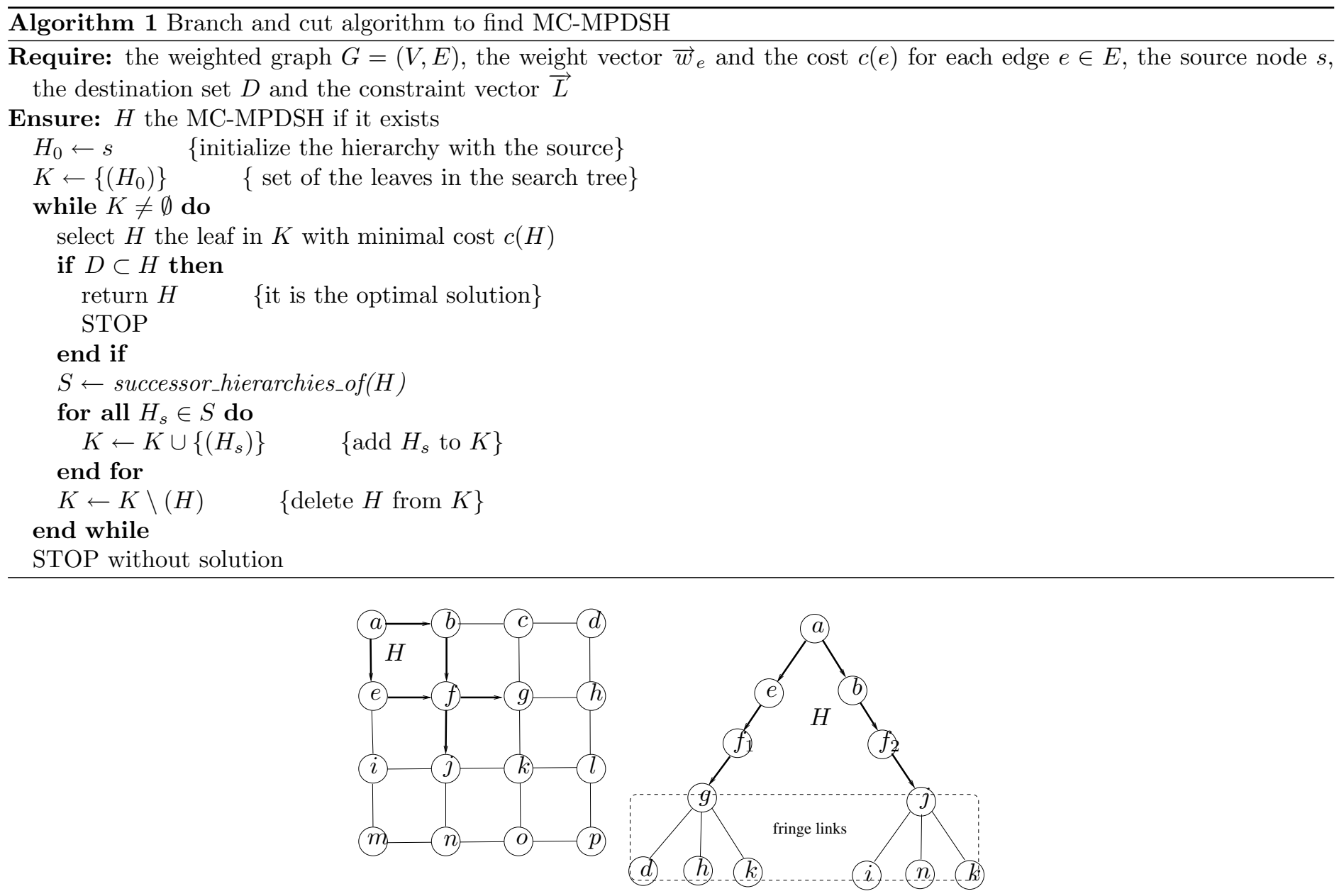

Figure 9: The fringe links of a hierarchy

Moreover, if a hierarchy $H$ is not valid, because the end-to-end constraints and/or the properties of the optimal hierarchy are not satisfied, adding a set of fringe links can not improve the hierarchy. Thus, the successors of a non valid hierarchy are non valid.

Properties 3, 4 and 6 can be directly applied to choose the valid continuation of the selected hierarchy. An arc leading to a node, which does not correspond to these properties is not useful for continuing the construction of the solution. For instance, let us suppose that a node $v$ is already present on the directed path $p(s, n)$, which connects the source to the start node $n$ of the arc $(n, v)$. According to Property 3, the optimal solution can not contain the path obtained by concatenating $p(s, n)$ and $p(n, v)$ : the arc $(n, v)$ is not a possible adjacent arc to continue the hierarchy $H$ construction toward obtaining the optimal solution. Moreover, the number of leaves as well as the number of the occurrences of an arbitrary graph node in the optimal solution are limited by $|D|$ as shown in Property 4, and a node can be branching node at most $\left\lfloor\frac{|D|}{2}\right\rfloor$ times as shown in Property 6). Only the fringe arcs corresponding to these bounds are useful.

To enumerate all of the valid successors of $H$ in the search tree, the subsets of the possible fringe arcs should be exhaustively enumerated. Moreover, this enumeration ensure that a hierarchy is visited only once by the branch and cut algorithm. The computation of the set of successors of a given hierarchy $H$ is described by Algorithm 2 .

Theorem 2. Algorithm 1 returns the optimal solution of the multi-constrained minimum partial spanning hierarchy problem if such a solution exists.

Proof. The algorithm enumerates all valid spanning hierarchies from the source node. Thus, the optimal solution is always visited before other valid solutions if such solutions exist as shown in Lemma 5. Let us suppose that another hierarchy with a lower cost is returned before the optimal solution. It is in contradiction with the fact that the optimal solution is the minimum cost solution of all feasible solutions. 


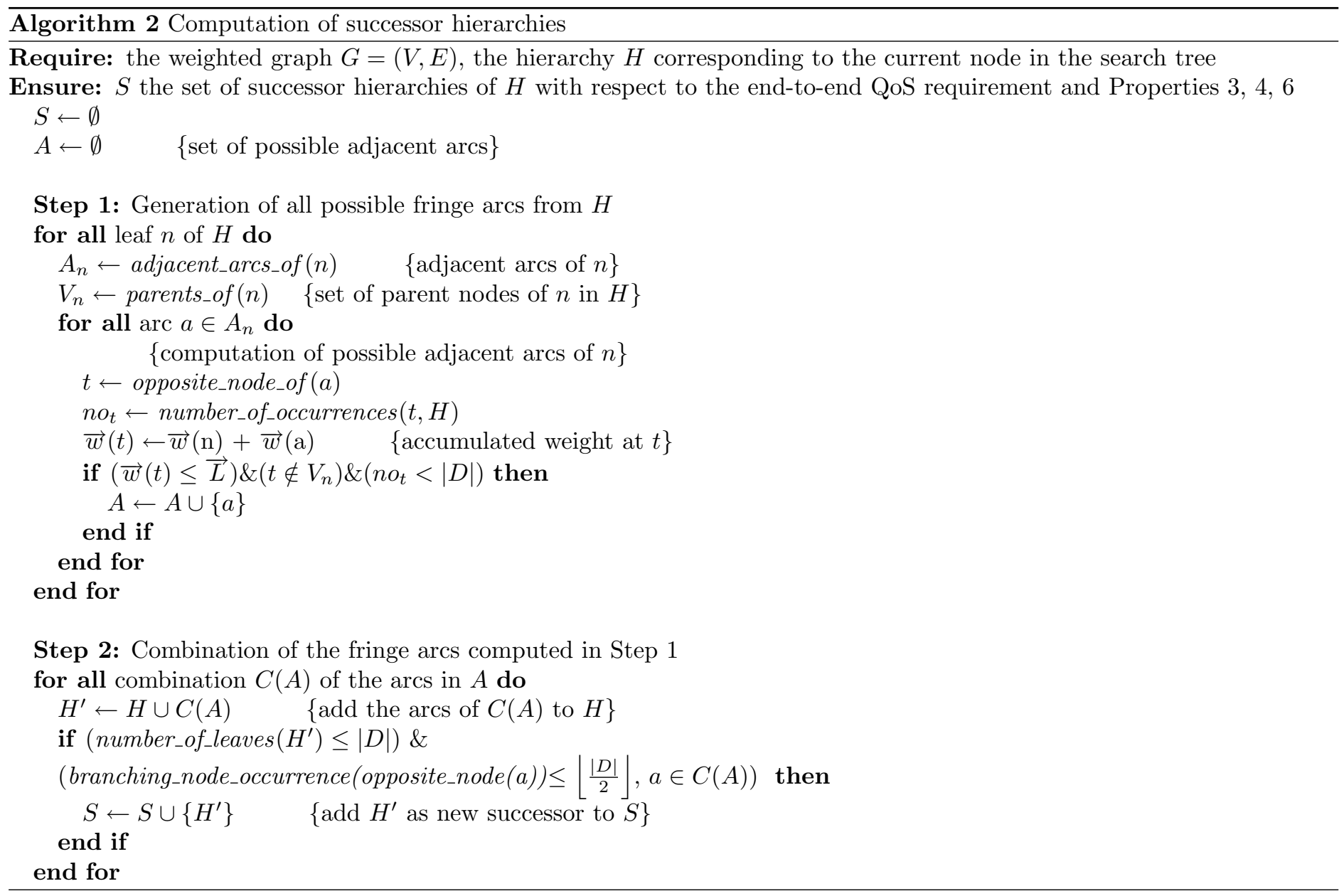




\section{Conclusions and Perspectives}

The main result of our investigations on the multi-constrained multicast routing problem is the characterization of the optimal solution structure. This structure always corresponds to a hierarchy. The construction of the minimum cost and the minimum non-linear length hierarchies are NP-difficult optimization problems. The algorithms computing heuristic solutions, like MAMCRA, manipulate hierarchies such as sets of paths rooted at the source and more or less redundancy-free spanning structures. In our paper, we proposed an initial study of the properties of the hierarchy-type solutions. Even if there is no simulation results in this paper, we argue that it is substantial to first identify the optimal solution structure of the addressed problem. An additional result of our analysis is that the optimal solution does not belong neither to the set of minimum cost paths nor to the set of shortest paths computed by using the frequently used non-linear length. Since hierarchies may contain several occurrences of a node and an edge, most of the enumeration algorithms are not appropriate to compute the optimal solution. Therefore, we proposed an efficient branch and cut algorithm to find the optimal hierarchy. Following this first study on multi-constrained minimum partial spanning hierarchies, important future work on exact and heuristic algorithms of this problem is needed to find efficient solutions.

\section{References}

[1] H. Lin and Z. Yu-Lin and R. Yong-Hong, Two multi-constrained multicast QoS routing algorithms, eighth ACIS International Conference on Software Engineering, Artificial Intelligence, Networking, and Parallel/Distributed Computing, 2007.

[2] V. Aggarwal and Y. P. Aneja and K. P. K. Nair,Minimal spanning tree subject to a side constraint, Journal of Computers \& Operations Research, volume 9(4), pp. 287-296, 1982.

[3] F. Bauer and A. Varma, ARIES: A Rearrangeable Inexpensive Edge-Based On-Line Steiner Algorithm, INFOCOM, pp. 361-368, 1996.

[4] Z. Wang and J. Crowcroft, Quality-of-Service Routing for Supporting Multimedia Applications, IEEE Journal of Selected Areas in Communications, volume 14(7), pp. 1228-1234, 1996.

[5] N. Ben Ali and M. Molnár and A. Belghith, Multi-constrained QoS Multicast Routing Optimization, IRISA, Research Report, number 1882, 2008.

[6] J. Crichigno and B. Baran, A Multicast Routing Algorithm using Multiobjective Optimization, ICT, Springer-Verlag, pp. $1107-1113,2004$.

[7] G. Feng, A multi-constrained multicast QoS routing algorithm, Journal of Comptuer Communications, volume 29, pp. 1811-1822, 2006.

[8] S. L. Hakimi, Steiner problem in graphs and its implications, Journal Networks, volume 1, pp. 113-133, 1971.

[9] S. P. Hong and S. J. Chung and B. H. Park, A fully polynomial bicriteria approximation scheme for the constrained spanning tree problem, Operations Research Letters, volume 32(3), 233-239, 2004.

[10] F. K. Hwang and D. S. Richards, Steiner Tree Problems, Journal of Networks, volume 22, pp. 55-89, 1992.

[11] P. V. Kompella and J. C. Pasquale and G. C. Polyzos, Multicast routing for multimedia communication, IEEE/ACM Transaction on Networks, volume 1(3), pp. 286-292, 1993.

[12] T. Korkmaz and M. Krunz, Multi-constrained optimal path selection, INFOCOM, pp. 834-843, 2001.

[13] F. A. Kuipers and P. Van Mieghem, MAMCRA: a constrained-based multicast routing algorithm, Journal of Computer Communications, volume 25(8), pp. 802-811, 2002.

[14] S. Kumar and P. Radoslavov and D. Thaler and C. Alaettinoglu and D. Estrin and M. Handley, The MASC/BGMP Architecture for Inter-Domain Multicast Routing, SIGCOMM, pp. 93-104, 1998.

[15] M. S. Levin and R. I. Nuiriakhmetov, Multicriteria Steiner Tree Problem for Communication Network, Information Technology in Engineering Systems, volume 9(3), pp. 199-209, 2009.

[16] X. Masip-Bruin and M. Yannuzzi and J. Domingo-Pascual and A. Fonte and M. Curado and E. Monteiro and F. Kuipers and P. Van Mieghem and S. Avallone and G. Ventre and P. Aranda-Gutierrez and M. Hollick and R. Steinmetz and L. Iannone and K. Salamatian, Research Challenges in QoS Routing, Computer Communications, volume 29(1), 2006. 
[17] P. Van Mieghem and F. A. Kuipers, Concepts of exact QoS routing algorithms, IEEE/ACM Transaction on Networks, volume 12(5), pp. 851-864, 2004.

[18] M. Molnár, Optimisation des communications multicast sous contraintes, University Rennes 1, 2008, http://ftp.irisa.fr/techreports/habilitations/molnar.pdf.

[19] D. Pinto and B. Barán and R. Fabregat, Multi-Objective Multicast Routing Based on Ant Colony Optimization, Proceeding of the 2005 Conference on Artificial Intelligence Research and Development, pp. 363-370, 2005.

[20] H. Takahashi and A. Matsuyama, An approximate solution for the Steiner problem in graphs, Journal of Mathematica Japonica, volume24(6), pp. 573-577, 1980.

[21] P. Van Mieghem and H. De Neve and A. F. Kuipers, Hop-by-hop Quality of Service Routing, Computer Networks, volume 37(3-4), pp. 407-423, 2001.

[22] D. Wang and F. Ergun and Z. Xu, Unicast and Multicast QoS Routing with Multiple Constraints, Quality of Service in Multiservice IP Networks, volume 3375, pp. 481-494, 2005.

[23] P. Winter, Steiner problem in networks: a survey, Journal of Networks, volume 17, pp. 129-167,1987.

[24] X. Zhang and J. Wei and C. Qiao, Constrained multicast routing in WDM networks with sparse light splitting, INFOCOM, volume 3, pp. 1781-1790, 2000.

[25] Z. Qing and P. Mehrdad and J. J. Garcia-Luna-Aceves, A Source-Based Algorithm for Delay-Constrained Minimum-Cost Multicasting, INFOCOM, pp. 377-385, 1995.

[26] R. C. Prim, Shortest connection networks and some generalisations, Bell System Technical Journal, volume 36, pp. 1389-1401, 1957. 